\title{
LA ORGANIZACIÓN DEL CURRÍCULO Y LA FORMACIÓN PROFESIONAL DE LOS EGRESADOS DEL AÑO 2009 DE LA FACULTAD DE EDUCACIÓN DE LA UNIVERSIDAD NACIONAL JORGE BASADRE GROHMANN DE TACNA
}

The Organization of Curriculum and Training of Graduates of 2009 of the Faculty of Education Jorge Basadre Grohmann National University of Tacna

Nayme Antonieta Choque Ticona ${ }^{3}$

\section{RESUMEN}

Objetivo: Determinar la influencia del nivel de la organización del currículo en el nivel de la formación profesional de los egresados del año 2009 de la Facultad de Educación de la Universidad Nacional Jorge Basadre Grohmann de Tacna.

Método: Es una investigación descriptiva que establece el nivel de la organización curricular y su influencia en el nivel de formación profesional. La muestra estuvo constituida por 62 egresados del año 2009 de la Facultad de Educación de la Universidad Nacional Jorge Basadre Grohmann de Tacna.

La recopilación de los datos se realizó a finales del año 2013 y a inicios del año 2014. Se elaboraron dos instrumentos: un cuestionario dirigido a los egresados del año 2009 y una ficha de análisis para establecer el nivel de organización del currículo.

Resultado: Los resultados de la investigación permitieron establecer que la organización del currículo es poco adecuada y el nivel de la formación profesional es regular. Estos resultados permitieron establecer la relación directa y significativa entre el nivel de la organización del currículo y

\footnotetext{
${ }^{3}$ Magister en Docencia Universitaria y Gestión Educativa
}

el nivel de la formación profesional de los egresados del año 2009 de la Facultad de Educación de la Universidad Nacional Jorge Basadre Grohmann de Tacna.

Conclusiones: La formación recibida en la Facultad de Ciencias de la Educación de la Universidad Nacional Jorge Basadre Grohmann, es regular según la percepción de los egresados, lo cual se evidencia en los resultados de la encuesta de opinión aplicada a los egresados del año 2009.

\section{PALABRAS CLAVES}

Currículo, Formación, Docente, Formación profesional, Organización del currículo

\section{ABSTRACT}

Objective: Determine the influence of the level of organization of the curriculum at the level of vocational training of graduates in 2009 from the Faculty of Education at the Jorge Basadre Grohmann National University of Tacna.

Method: A descriptive research that establishes the level of curricular organization and its influence on the level of professional training. The sample consisted of 62 graduates in 2009 from the Faculty of Education at the Jorge Basadre Grohmann National University of Tacna.

The data collection was conducted in late 2013 and early 2014. Two instruments were developed: a 
questionnaire to the graduates of 2009, and a data-analysis to establish the level of organization of the curriculum.

Result: The results of the research allowed to establish that the organization of the curriculum is inadequate and the level of training is regular. These results allowed to establish direct and significant relationship between the level of organization of the curriculum and the level of training of graduates in 2009 from the Faculty of Education at the
Jorge Basadre Grohmann National University of Tacna.

Conclusions: The training received in the Faculty of Education at the National University Jorge Basadre Grohmann, is regulated according to the perception of the graduates, which is evidenced by the results of the opinion poll applied to graduates of 2009.

\section{KEYWORDS}

Curriculum, Education, Teaching, Vocational training, curriculum Organization

\section{INTRODUCCIÓN}

La sociedad demanda de buenos profesionales para formar integralmente a los futuros ciudadanos de nuestro país desde el nivel inicial hasta el nivel secundaria.

Una adecuada organización del currículo de las carreras profesionales de educación de las universidades de nuestro país permitirá asegurar buenos profesionales en educación. Por ende los estudiantes recibirán un servicio de calidad que será evidente cuando el egresado de secundaria se inserte en la sociedad satisfactoriamente.

Es evidente la importancia de formar buenos profesionales en educación para que esto redunde en la formación de los estudiantes de la educación básica regular.

En nuestro país, los institutos pedagógicos y las universidades a través de las especialidades y facultades de educación, se encargan de formar a los futuros docentes, quienes en el ejercicio de sus funciones formarán a los futuros ciudadanos que demanda la sociedad.

Para cumplir con las exigencias de la sociedad, a finales del 2006 el Ministerio de Educación (MED) inició procesos de evaluación a los docentes de todo el país, dónde se obtuvo resultados no satisfactorios lo que lleva a la reflexión sobre la calidad de los docentes, específicamente en su formación.

Ésta situación necesitó de urgentes e inmediatas medidas para solucionarla, como por ejemplo programas de capacitación y evaluaciones para procesos de nombramiento y contrato, que buscaba garantizar docentes de calidad. Actualmente la Dirección Sectorial de Educación de Tacna, lleva a cabo los procesos de contrato, a través de exámenes de acuerdo a la matriz de evaluación que emite el Ministerio de Educación.

Luego de un análisis de la problemática planteada, se llega a establecer algunas posibles causas que lo estén generando. Entre ellas se puede considerar a las políticas educativas que deberían garantizar una formación docente de calidad, tal es el caso la acreditación de las carreras profesionales de educación. Instituciones superiores que no brindan un servicio de calidad, por no contar con el financiamiento 
que permite una infraestructura y equipamiento adecuado y suficiente; otro aspecto es la organización del currículo que debería ajustarse a la realidad y a las exigencias de la sociedad actual.

Se considera la última causa, la organización del currículo, como la de mayor importancia en la formación profesional del futuro docente, de allí que se plantea la intención de investigar al currículo y a la formación profesional.

La formación profesional de docentes se da tanto en universidades como en institutos superiores pedagógicos, la admisión a estos centros se realiza mediante concurso público. De acuerdo con el Artículo $21^{\circ}$ de la Ley Universitaria $\mathrm{N}^{\circ} 23733$, la admisión a la universidad se realiza mediante concurso, con las excepciones previstas en el Art. 56 de la misma ley, una o dos veces cada año durante los períodos de vacaciones. El estatuto de la universidad y los reglamentos de las facultades de educación establecen los mecanismos que permiten evaluar los intereses vocacionales, las aptitudes y los rasgos de personalidad para el estudio de la carrera. La universidad establece con la debida anticipación el número de vacantes, cuya cifra es inmodificable después de ser aprobada y publicada para cada concurso.

Teniendo en cuenta el protagonismo del docente en el sistema educativo, es necesario ponerle mucha atención a su formación profesional. Formación que requiere un análisis en sus dos etapas: la formación inicial y la formación en servicio. Ambas etapas son esenciales para asegurar un alto nivel de desempeño.

La Formación en servicio es el complemento de la formación inicial a través de cursos, diplomados, actualizaciones y especializaciones. Y la formación inicial se da en diez ciclos académicos en las instituciones superiores y se hace evidente en el desempeño de sus funciones.

Para el logro de un alto nivel de desempeño, es necesario entender que la organización del currículo es base fundamental e influye en la formación profesional. La organización del currículo debemos considerarla como un sistema en el que entra en juego una serie de elementos que interactúan y se concreta en el logro del Perfil profesional.

\section{MATERIALES Y MÉTODOS}

El tipo de investigación es Básica. Se trata de un diseño descriptivo. Que busca profundizar el conocimiento de la relación del nivel de la organización del currículo y el nivel de la formación profesional de los egresados del año 2009 de la Facultad de Ciencias de la Educación de la Universidad Nacional Jorge Basadre Grohmann de Tacna.

Las unidades de estudio los constituyeron los Egresados de la Facultad de Ciencias de la Educación, quienes pertenecen a las especialidades de Ciencias Sociales y Promoción Socio Cultural, Lengua Literatura y Gestión Educativa, Matemática, Computación e Informática, Ciencias de la Naturaleza, Tecnología y Ambiente, Idioma Extranjero, Traductor e Intérprete.

La población estuvo constituida por 96 Egresados del año 2009, quienes pertenecen a las diferentes especialidades de la Facultad de Ciencias de la Educación de la 
UNJBG. La muestra estuvo constituida por 62 egresados del año 2009, seleccionados por criterio.

Se trabajó con la técnica análisis documental para establecer el nivel de la organización curricular. Asimismo, se trabajó se utilizó la técnica de la encuesta para establecer el nivel de formación profesional de los egresados del año 2009 de la Facultad de Ciencias de la Educación de la Universidad Nacional Jorge Basadre Grohmann de Tacna.

Se aplicó un cuestionario a los egresados del año 2009 de la Facultad de Ciencias de la Educación de la Universidad Nacional Jorge Basadre Grohmann de Tacna para establecer el nivel de formación profesional.

\section{RESULTADOS}

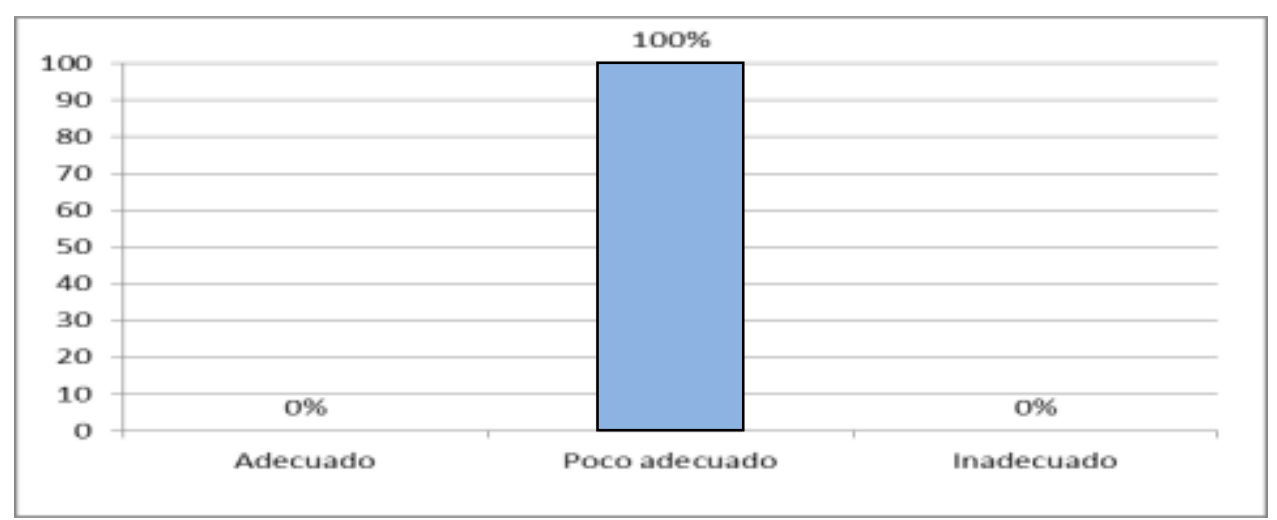

Fuente: Tabla $N^{\circ} 05$

\section{Figura № 1}

\section{Nivel de organización del currículo}

La figura $N^{\circ} 1$ se presenta información sobre el nivel de la organización del currículo.

En ella se observa que el $100 \%$ de los expertos manifiestan que la organización del currículo del año 2009 de la Facultad de Ciencias de la Educación de la UNJBG es poco adecuado.

Los resultados obtenidos permiten afirmar que el nivel de organización curricular del currículo del año 2009 de la Facultad de Ciencias de la Educación de la UNJBG es poco adecuado.

De las cuatro dimensiones solo se cumple con la dimensión viabilidad de la propuesta curricular. La totalidad de los evaluadores coinciden que la coherencia de la propuesta curricular y la evaluación de los resultados del diseño y desarrollo curricular, no se cumplen. Y la dimensión de integración entre los distintos organizadores curriculares se cumple en forma parcial.

Finalmente, los factores que determinan que el nivel de la organización del currículo sea poco adecuado son: La coherencia de la propuesta curricular (100\% nada coherente) y la evaluación de los resultados del diseño y desarrollo curricular (100\% resultados de bajo impacto). 


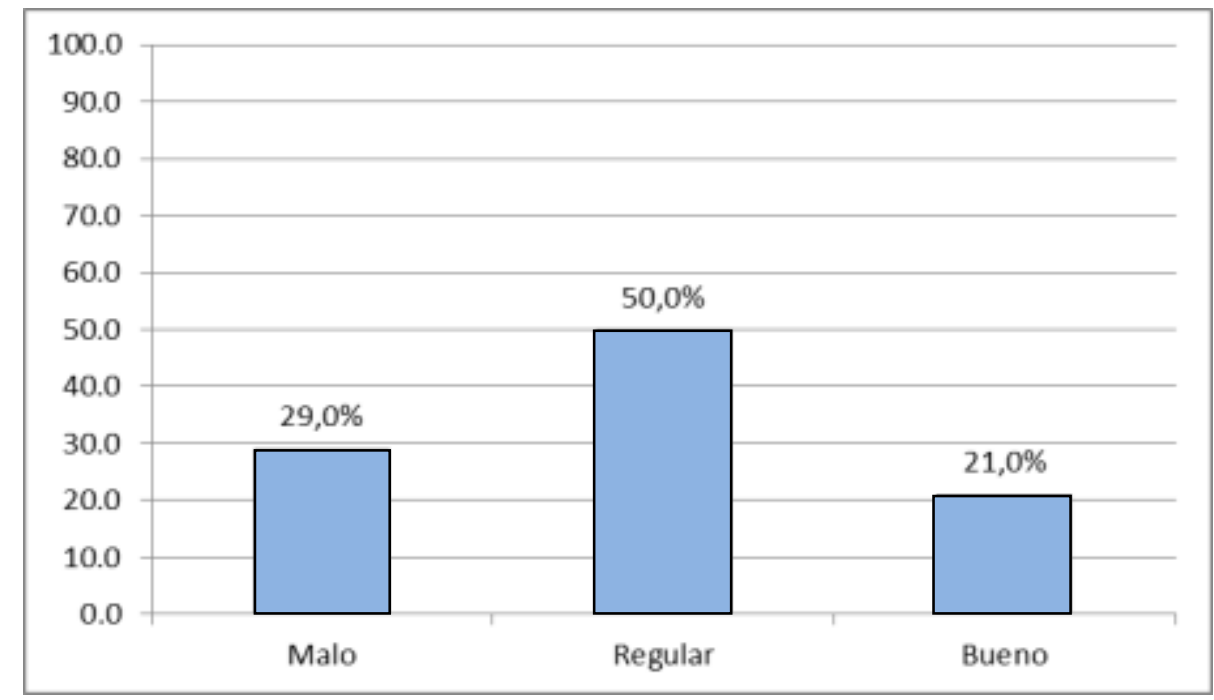

Fuente: Tabla $\mathrm{N}^{\circ} 13$

Figura № 2

Aspecto

Formación Pedagógica

En la Figura $N^{\circ} 2$ se presenta información sobre el Aspecto formación pedagógica del Perfil Profesional General de la Facultad de Ciencias de la Educación de la UNJBG.

Se observa que el $50,0 \%$ del total de los egresados encuestados afirman que la formación recibida en el aspecto formación pedagógica es regular, el 29,0\% del total indican que fue mala la formación recibida en el aspecto formación pedagógica y solo el $21,0 \%$ del total manifiesta que fue buena la formación recibida en el aspecto formación pedagógica.

De acuerdo a la percepción de los egresados encuestados (50\%), el aspecto formación pedagógica es regular, información relevante porque dicho aspecto es sumamente importante para desempeñarse en el mercado laboral educativo.

Todas las características del aspecto formación pedagógica del perfil general del egresado se desarrollaron con deficiencias:

- Analiza, describe y explica la problemática educacional.

- Investiga el proceso educativo

- Planifica la actividad educativa

- Realiza la ejecución, organización y administración de instituciones y proyectos educacionales.

- Conduce el proceso de enseñanza-aprendizaje

- Elabora y aplica los materiales educativos

- Promueve y desarrolla la comunidad local, regional y nacional

- Evalúa el proceso educativo 


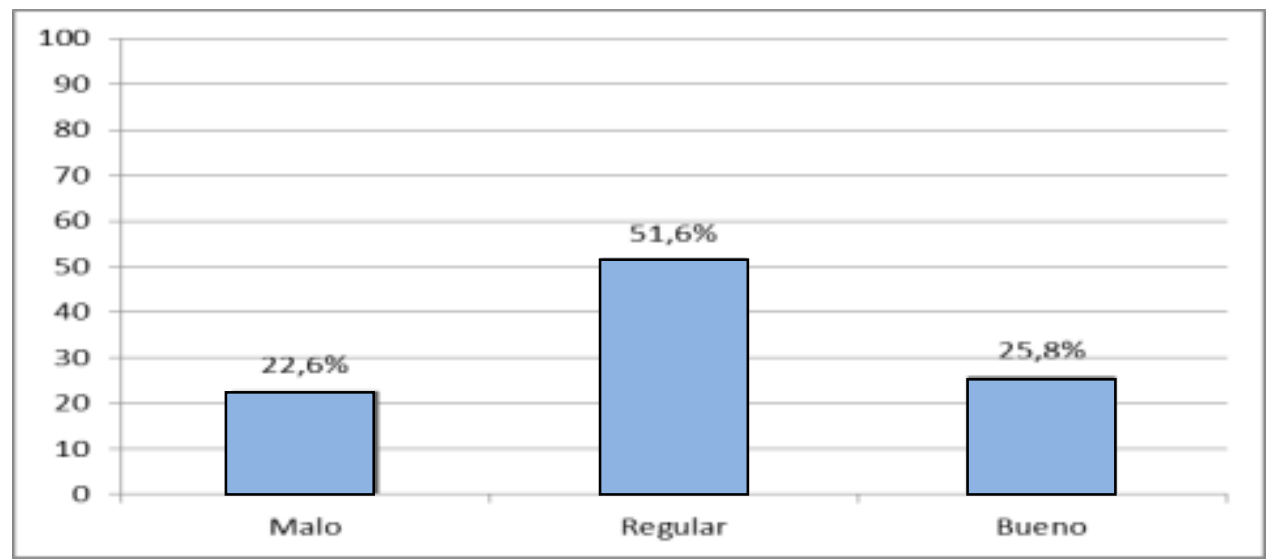

Fuente: Tabla $\mathrm{N}^{\circ} 17$

Figura № 3

Nivel de Formación Profesional alcanzado según la percepción de los egresados del 2009 de la Facultad de Ciencias de la Educación - UNJBG

En la Figura $\mathrm{N}^{\circ} 3$, se presenta la información general sobre la Formación Profesional según la percepción de los egresados de la Facultad de Ciencias de la Educación de la UNJBG.

Se observa que el $51,6 \%$ del total de los egresados encuestados afirman que la formación profesional recibida fue regular, el $25,8 \%$ del total de egresados encuestados indican que fue buena la formación profesional recibida y el $22,6 \%$ del total de egresados encuestados manifiestan que fue mala la formación profesional recibida.

Basado en la información presentada se concluye que más del $50 \%$ de los egresados encuestados manifiestan que la formación profesional recibida teniendo en cuenta el perfil general del egresado de la Facultad de Ciencias de la Educación de la UNJBG es regular. Dicho resultado es evidente porque existen limitaciones en el desarrollo de los siete aspectos.

Finalmente, los factores que determinan que el nivel de formación profesional sea regular son: Formación pedagógica, prácticas pre profesionales, actividades cocurriculares y consejería.

\section{Relación entre variables}

Coherencia de la propuesta curricular

\begin{tabular}{|c|c|c|c|}
\hline & Rango & f & $\%$ \\
\hline Coherente & $8-11$ & 0 & 0 \\
\hline Poco coherente & $4-7$ & 0 & 0 \\
\hline Nada coherente & $0-3$ & 4 & 100 \\
\hline \multicolumn{2}{|c|}{ TOTAL } & 4 & 100 \\
\hline
\end{tabular}

Formación Profesional

\begin{tabular}{|c|c|c|}
\hline Nivel & $f$ & $\%$ \\
\hline Buena & 16 & 25,8 \\
\hline Regular & 32 & 51,6 \\
\hline Mala & 14 & 22,6 \\
\hline Total & 62 & 100,0 \\
\hline
\end{tabular}


La dimensión coherencia de la propuesta curricular es nada coherente, resultado que se encuentra en relación con el nivel regular de la formación profesional.

\begin{tabular}{|c|c|c|c|}
\hline \multicolumn{4}{|c|}{$\begin{array}{l}\text { La integración entre los distintos } \\
\text { organizadores curriculares }\end{array}$} \\
\hline & Rango & $\mathbf{f}$ & $\%$ \\
\hline Relación & $12-17$ & 3 & 75 \\
\hline Poca relación & $6-11$ & 1 & 25 \\
\hline Nada de relación & $0-5$ & 0 & 0 \\
\hline \multicolumn{2}{|c|}{ TOTAL } & 4 & 100 \\
\hline
\end{tabular}

\begin{tabular}{lcr}
\multicolumn{3}{c}{ Formación Profesional } \\
\hline \multicolumn{1}{c}{ Nivel } & $\mathbf{f}$ & \multicolumn{1}{c}{$\%$} \\
\hline Buena & 16 & 25,8 \\
Regular & $\mathbf{3 2}$ & $\mathbf{5 1 , 6}$ \\
Mala & 14 & 22,6 \\
\multicolumn{1}{c}{ Total } & $\mathbf{6 2}$ & $\mathbf{1 0 0 , 0}$ \\
\hline Fuente: Cuestionario aplicado a los Egresados del año \\
2009 de la Facultad de Ciencias de la Educación de la \\
UNJBG
\end{tabular}

La dimensión integración entre los distintos organizadores curriculares es parcial, resultado que se encuentra en relación con el nivel regular de la formación profesional.

La evaluación de los resultados del diseño y desarrollo curricular

\begin{tabular}{lccr}
\hline & Rango & f & \% \\
\hline $\begin{array}{l}\text { Resultados de alto } \\
\text { impacto }\end{array}$ & $4-5$ & 0 & 0 \\
$\begin{array}{l}\text { Resultados de impacto } \\
\text { moderado }\end{array}$ & $2-3$ & 0 & 0 \\
$\begin{array}{l}\text { Resultados de bajo } \\
\text { impacto }\end{array}$ & $\mathbf{0 - 1}$ & $\mathbf{4}$ & $\mathbf{1 0 0}$ \\
\multicolumn{1}{c}{ TOTAL } & & $\mathbf{4}$ & $\mathbf{1 0 0}$ \\
\hline \begin{tabular}{l} 
Fuente: Ficha de Análisis de la Organización curricular \\
\hline
\end{tabular}
\end{tabular}

Formación Profesional

\begin{tabular}{lcr}
\hline \multicolumn{1}{c}{ Nivel } & $\mathbf{f}$ & \multicolumn{1}{c}{$\%$} \\
\hline Buena & 16 & 25,8 \\
Regular & $\mathbf{3 2}$ & $\mathbf{5 1 , 6}$ \\
Mala & 14 & 22,6 \\
\multicolumn{1}{r}{ Total } & $\mathbf{6 2}$ & $\mathbf{1 0 0 , 0}$ \\
\hline Fuente: Cuestionario aplicado a los Egresados del año \\
2009 de la Facultad de Ciencias de la Educación de la \\
UNJBG
\end{tabular}

La dimensión evaluación de los resultados del diseño y desarrollo curricular es de resultados de bajo impacto, resultado que se encuentra en relación con el nivel regular de la formación profesional.

NIVEL DE LA ORGANIZACIÓN DEL CURRÍCULO

\begin{tabular}{|c|c|c|}
\hline Nivel & $f$ & $\%$ \\
\hline Adecuado & 0 & 0 \\
\hline Poco adecuado & 4 & 100 \\
\hline Inadecuado & 0 & 0 \\
\hline TOTAL & 4 & 100 \\
\hline
\end{tabular}

\section{NIVEL DE FORMACIÓN PROFESIONAL}

\begin{tabular}{lcr}
\multicolumn{1}{c}{ Nivel } & Frecuencia & Porcentaje \\
\hline Buena & 16 & 25,8 \\
Regular & $\mathbf{3 2}$ & $\mathbf{5 1 , 6}$ \\
Mala & 14 & 22,6 \\
$\quad$ Total & $\mathbf{6 2}$ & $\mathbf{1 0 0 , 0}$ \\
\hline
\end{tabular}

Fuente: Cuestionario aplicado a los Egresados del año 2009 de la Facultad de Ciencias de la Educación de la UNJBG

Se observa que el nivel de la organización del currículo es poco adecuado y el nivel de la formación profesional es regular. Por lo que se afirma que existe una relación directa y significativa entre el nivel de la organización del currículo y el 
nivel de la formación profesional. Lo que nos permite afirmar que una adecuada organización del currículo permitirá una buena formación profesional.

COEFICIENTE DE CORRELACIÓN DE RHO DE SPEARMAN

\begin{tabular}{|c|c|c|c|c|}
\hline & FP & OC & D & D2 \\
\hline $\mathbf{1}$ & 3 & 2.5 & -0.5 & 0.25 \\
\hline $\mathbf{2}$ & 1 & 1 & 0 & 0 \\
\hline $\mathbf{3}$ & 2 & 2.5 & 0.5 & 0.25 \\
\hline
\end{tabular}

$\mathrm{FP}=$ Formación Profesional

$\mathrm{OC}=$ Organización del currículo

$\rho=1-\frac{6 \sum D^{2}}{N\left(N^{2}-1\right)}$

\section{Dónde:}

$D=$ Es la diferencia entre los correspondientes estadísticos de orden de $\mathrm{x}-\mathrm{y}$.

$\mathrm{N}=$ Es el número de parejas.

$\rho=1-\frac{6(0.5)}{3\left(3^{2}-1\right)}=1-\frac{3}{3(9-1)}=1-\frac{3}{3(8)}=1-\frac{3}{24}=1-0.125=0.875$

$\rho=0.875$

$>0.7=$ Relación significativa

\section{NIVEL DE INFLUENCIA}

$r^{2}=\rho^{2} \times 100$

$r^{2}=(0.875)^{2} \times 100$

$r^{2}=0.77 * 100$

$r^{2}=77 \%$

Existe un $77 \%$ de influencia.

\section{CONCLUSIONES}

a) El nivel de organización del currículo vigente en el año 2009 de la Facultad de Ciencias de la Educación de la Universidad Nacional Jorge Basadre Grohmann, es poco adecuado según los resultados obtenidos a través de la ficha de análisis.

b) La formación recibida en la Facultad de Ciencias de la Educación de la Universidad Nacional Jorge Basadre Grohmann, es regular según la 
percepción de los egresados, lo cual se evidencia en los resultados de la encuesta de opinión aplicada a los egresados del año 2009.

c) Se aprecia una relación directa y significativa entre el nivel poco adecuado de la organización del currículo y el nivel regular de formación profesional de los egresados del año 2009 de la Facultad de Educación de la Universidad Nacional Jorge Basadre Grohmann de Tacna.

d) El nivel de organización del currículo vigente en el año 2009 de la Facultad de Ciencias de la Educación de la Universidad Nacional Jorge Basadre Grohmann influyó en forma significativa en el nivel de percepción que tienen los egresados sobre la formación profesional recibida.

\section{REFERENCIAS BIBLIOGRÁFICAS}

- $\quad$ ARNAZ, J. A. (1981). La planeación curricular. México: Trillas

- DÍAZ-Barriga, F., Lule, M. L., Pacheco, D., Rojas-Drummond, S., Saad, E. (1990). Metodología de Diseño curricular para educación superior. México: Trillas

- CATACORA L. (2014). Criterios para redactar y divulgar estudios y trabajos de investigación científica. Tacna. Universidad Privada de Tacna.

- CUENCA R. \& Stojnic L. (Eds.). (2008). La cuestión docente Perú: carrera pública magisterial y el discurso del desarrollo profesional. Buenos Aires.

- DÍAZ Barriga, Ángel. (1990) Ensayos sobre la problemática curricular. México: Trillas: ANUIES, (reimpresión 1992)

- De Los A., M. (2006). Aprender y Enseñar en Contextos Complejos Multiculturalidad, Diversidad y Fragmentación. Perú.

- IBARRA O. (1970). Didáctica moderna. Venezuela.

- LEY UNIVERSITARIA N²3733

- SALAS P. (2013). Cultura pedagógica. Perú.

- TOBÓN S. (2005). Formación basado en competencias. Ecoe Ediciones Ltda. Colombia.

- VAldiVIA R. (2009). Elaborando la Tesis (una propuesta). Tacna. Universidad Privada de Tacna.

Recibido: 26/5/2015

Aceptado para publicación: $6 / 7 / 2015$ 